\title{
A Survey on Wireless Position Estimation
}

\author{
Sinan Gezici
}

Published online: 2 October 2007

(C) Springer Science+Business Media, LLC. 2007

\begin{abstract}
In this paper, an overview of various algorithms for wireless position estimation is presented. Although the position of a node in a wireless network can be estimated directly from the signals traveling between that node and a number of reference nodes, it is more practical to estimate a set of signal parameters first, and then to obtain the final position estimation using those estimated parameters. In the first step of such a two-step positioning algorithm, various signal parameters such as time of arrival, angle of arrival or signal strength are estimated. In the second step, mapping, geometric or statistical approaches are commonly employed. In addition to various positioning algorithms, theoretical limits on their estimation accuracy are also presented in terms of Cramer-Rao lower bounds.
\end{abstract}

Keywords Position estimation - Cramer-Rao lower bound - Mapping techniques · Bayerian estimation · Maximum likelihood estimation

\section{Introduction}

Recently, the subject of positioning in wireless networks has drawn considerable attention. With accurate position estimation, a variety of applications and services such as enhanced911, improved fraud detection, location sensitive billing, intelligent transport systems, and improved traffic management can become feasible for cellular networks [1]. For short-range networks, on the other hand, position estimation facilitates applications such as inventory tracking, intruder detection, tracking of fire-fighters and miners, home automation and patient monitoring [2]. These potential applications of wireless positioning have also been recognized by the IEEE, which set up a standardization group 802.15.4a for designing a new physical layer for low-data rate communications combined with positioning capabilities [3]. Also, the Federal Communications Commission (FCC) in the US has required wireless providers to locate mobile users within tens of meters for emergency 911 calls [4].

S. Gezici $(\bowtie)$

Department of Electrical and Electronics Engineering, Bilkent University, Bilkent, Ankara 06800, Turkey e-mail: gezici@ee.bilkent.edu.tr 
(a)

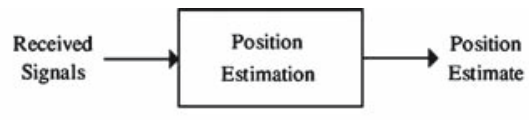

(b)

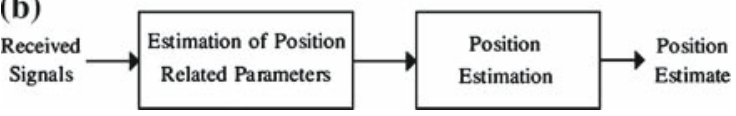

Fig. 1 a Direct positioning, b two-step positioning

In order to realize potential applications of wireless positioning, accurate estimation of position should be performed even in challenging environments with multipath and non-lineof-sight (NLOS) propagation [5]. For accurate position estimation, the details of the position estimation process and its theoretical limits should be well-understood. Position estimation can be defined as the process of estimating the position of a node, ${ }^{1}$ called the "target" node, in a wireless network by exchanging signals between the target node and a number of reference nodes. ${ }^{2}$ The position of the target node can be estimated by the target node itself, which is called self-positioning, or it can be estimated by a central unit that obtains information via the reference nodes, which is called remote-positioning (network-centric positioning) [6]. Also, depending on whether the position is estimated from the signals traveling between the nodes directly or not, two different position estimation schemes can be considered. Direct positioning refers to the case in which the position estimation is performed directly from the signals traveling between the nodes [7]. On the other hand, two-step positioning extracts certain signal parameters from the signals first, and then estimates the position based on those signal parameters (Fig. 1). Although the two-step positioning is suboptimal in general, it can have significantly lower complexity than the direct approach. Also, the performance of the two approaches are usually very close for sufficiently high signal bandwidths and/or signalto-noise ratios (SNRs) [7,8]. Therefore, the two-step positioning is the common technique in most positioning systems, which is the main focus of this paper.

In the first step of a two-step positioning algorithm, signal parameters, such as time-ofarrival (TOA) and received signal strength (RSS), are estimated. Then, in the second step, the position of the target node is estimated based on the signal parameters obtained in the first step, as shown in Fig. 1b. In the second step of position estimation, mapping (fingerprinting) approaches, geometric or statistical techniques can be used depending on the accuracy requirements and system constraints.

The remainder of the paper is organized as follows. In Sect. 2, estimation of position related signal parameters is studied, and RSS, angle-of-arrival (AOA), TOA, time-difference-ofarrival (TDOA), and other parameter estimation schemes are investigated. Then, in Sect.3, position estimation schemes based on mapping, geometric and statistical approaches are studied, and theoretical limits are presented in terms of Cramer-Rao lower bounds (CRLBs). Finally, some concluding remarks are made in Sect. 4.

\section{Estimation of Position Related Parameters}

In the first step of a two-step positioning algorithm, position related parameters of the signals traveling between the target node and a number of reference nodes are estimated. For

\footnotetext{
1 A "node" refers to any device involved in the position estimation process, such as a cellular phone, a base station or a wireless sensor.

2 In this article, radiolocation is considered, which is the process of position estimation through the use of radio signals. Other techniques for position estimation include dead-reckoning and proximity systems [1].
} 


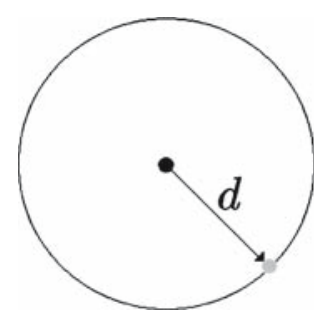

Fig. 2 One node measures the RSS and determines the distance $d$ between itself and the other node, which defines a circle of uncertainty

self-positioning, the signals received by the target node are used by the target node itself for parameter estimation. On the other hand, for remote-positioning, each reference node can estimate the parameter(s) of the signal it receives from the target node, and forward its estimate to a central unit. ${ }^{3}$ In other words, the parameter estimation block in Fig. $1 \mathrm{~b}$ resides in the target node for self-positioning systems and in the reference nodes, with each node estimating a subset of the total signal parameters, for remote-positioning systems.

Depending on accuracy requirements and system constraints, various signal parameters can be estimated in the first-step of a positioning algorithm. Commonly, signal parameters employed in positioning are related to power, direction and/or timing of a received signal.

\subsection{Received Signal Strength (RSS)}

The power, or energy, of a signal traveling between two nodes is a signal parameter that contains information related to the distance ("range") between those nodes. This parameter, commonly referred to as RSS, can be used together with a path-loss and shadowing model to provide a distance estimate. Therefore, in the error-free case, an RSS estimate at a node determines the position of the other node on a circle for two-dimensional positioning, ${ }^{4}$ as shown in Fig. 2.

A signal traveling from one node to another experiences fast (multipath) fading, shadowing and path-loss [9]. Ideally, averaging RSS over a sufficiently long time interval excludes the effects of multipath fading and shadowing, which results in the following model: ${ }^{5}$

$$
\bar{P}(d)=P_{0}-10 n \log _{10}\left(d / d_{0}\right),
$$

where $n$ is the path loss exponent, $\bar{P}(d)$ the average received power in $\mathrm{dB}$ at a distance $d$, and $P_{0}$ is the received power in $\mathrm{dB}$ at a short reference distance $d_{0}$.

In practice, the observation interval is not long enough to mitigate the effects of shadowing. Therefore, the received power is commonly modeled to include both path-loss and shadowing effects, the latter of which are modeled as a zero mean Gaussian random variable with a variance of $\sigma_{\mathrm{sh}}^{2}$ in the logarithmic scale. Therefore, the received power $P(d)$ in $\mathrm{dB}$ can be expressed as

$$
P(d) \sim \mathcal{N}\left(\bar{P}(d), \sigma_{\mathrm{sh}}^{2}\right)
$$

\footnotetext{
3 It is also possible to forward the received signals directly to the central unit, and to perform both parameter and position estimation there. However, this approach has considerably higher complexity and is not commonly preferred for two-step positioning systems. However, for direct-positioning systems, that is the only way to perform remote positioning.

4 Two-dimensional positioning is considered in this paper for the convenience of illustrations.

5 Note that there is also thermal noise in real systems, which is commonly position-dependent. It is assumed that the effects of thermal noise are sufficiently mitigated [10].
} 


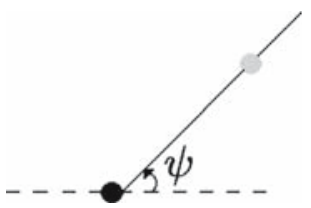

Fig. 3 AOA measurement between two nodes

where $\bar{P}(d)$ is as given in (1). Note that this model can be used in both line-of-sight (LOS) and NLOS scenarios with an appropriate choice of channel parameters.

From the received power model in (2), the CRLB for unbiased distance estimators can be expressed as [10]

$$
\sqrt{\operatorname{Var}\{\hat{d}\}} \geq \frac{(\ln 10) \sigma_{\mathrm{sh}} d}{10 n}
$$

where $\hat{d}$ represents an unbiased estimate for the distance $d$. From (3), it is observed that the RSS estimates get more accurate as the standard deviation of the shadowing decreases, since RSS estimates vary less around the true average power in that case. Also a larger path-loss exponent results in a smaller lower bound, as the average power becomes more sensitive to distance for larger $n$. Finally, the accuracy deteriorates as the distance between the nodes increases.

\subsection{Angle of Arrival (AOA)}

The angle between two nodes can be determined by estimating the AOA parameter of a signal traveling between the nodes (Fig. 3). Commonly, antenna arrays are employed in order to estimate the AOA of a signal. ${ }^{6}$ The main principle behind the AOA estimation via antenna arrays is that differences in arrival times of an incoming signal at different antenna elements include the angle information if the array geometry is known.

For narrowband signals, time differences can be represented as phase shifts. Therefore, the combinations of the phase shifted versions of received signals at different array elements can be tested in order to estimate the AOA [1]. However, for wideband systems, time delayed versions of received signals should be considered, since a time delay cannot be represented by a unique phase value for a wideband signal.

In order to study the effects of system parameters on the achievable accuracy of an AOA estimator, consider a uniform linear array (ULA) with $N_{\mathrm{a}}$ elements and assume the same fading coefficient $\alpha$ for all signals arriving at the array elements. Then, the CRLB on the variance of unbiased AOA estimators can be expressed as $[12,13]$

$$
\sqrt{\operatorname{Var}\{\hat{\psi}\}} \geq \frac{\sqrt{3} c}{\sqrt{2} \pi \sqrt{\operatorname{SNR}} \beta \Delta \sqrt{N_{\mathrm{a}}\left(N_{\mathrm{a}}^{2}-1\right)} \sin \psi},
$$

where $\psi$ is the AOA, $c$ the speed of light, $\mathrm{SNR}=\alpha^{2} E / \mathcal{N}_{0}$ is the signal-to-noise ratio for each element, with $E$ denoting the signal energy and $\mathcal{N}_{0}$ being the spectral density of background

6 Another approach is to use the ratio of RSS estimates between at least two directional antennas located on a node [11]. 
noise, ${ }^{7} \Delta$ the inter-element spacing, and $\beta$ is the effective bandwidth defined by

$$
\beta=\left(\frac{1}{E} \int_{-\infty}^{\infty} f^{2}|S(f)|^{2} \mathrm{~d} f\right)^{1 / 2}
$$

with $S(f)$ representing the Fourier transform of the received signal.

From (4), it is observed that as the SNR, effective bandwidth, inter-element spacing and/or the number of antenna elements is increased, the accuracy of AOA estimation increases. It is also noted that a ULA provides the best AOA estimation accuracy when the signal direction and the ULA line are perpendicular to each other.

\subsection{Time of Arrival (TOA)}

Similar to the RSS parameter, estimating the flight time of a signal traveling from one node to another, called TOA, provides information related to the distance between those two nodes. Therefore, in the absence of any errors, a TOA estimate provides an uncertainty region in the shape of a circle as shown in Fig. 2.

In order to calculate the TOA parameter for a signal traveling between two nodes, the nodes must either have a common clock, or exchange timing information by certain protocols such as a two-way ranging protocol $[3,14,15]$.

Conventionally, TOA estimation is performed via correlator or matched filter (MF) receivers [16]. Consider a scenario in which $s(t)$ is transmitted from a node to another, and the received signal is expressed as

$$
r(t)=s(t-\tau)+n(t),
$$

where $\tau$ represents the TOA and $n(t)$ is white Gaussian noise with zero mean and a spectral density of $\mathcal{N}_{0} / 2$. Then, the correlator-based approach correlates the received signal with a local template $s(t-\hat{\tau})$ for various delays $\hat{\tau}$, and calculates the delay corresponding to the correlation peak. Similarly, the MF approach employs a filter that is matched to the transmitted signal and estimates the instant at which the filter output reaches its largest value. Both approaches are optimal in the maximum likelihood (ML) sense for the signal model in (6). However, in practical systems, the signal arrives at the receiver via multiple signal paths. In such multipath environments, the conventional schemes become suboptimal as they use the transmitted signal to set their template signals or MF impulse responses. ${ }^{8}$ In order to obtain accurate TOA estimation in multipath environments, high-resolution time delay estimation techniques, such as that described in [17], have been studied for narrowband systems, and first path detection algorithms are proposed for ultra-wideband (UWB) systems [14,18-20].

In order to observe the main relations between the signal bandwidth and the theoretical limits for TOA estimation, consider the CRLB for the signal model in (6), which is given by Poor [21], Cook and Bernfeld [22] ${ }^{9}$

$$
\sqrt{\operatorname{Var}(\hat{\tau})} \geq \frac{1}{2 \sqrt{2} \pi \sqrt{\operatorname{SNR} \beta}},
$$

where $\hat{\tau}$ represents an unbiased TOA estimate, $\mathrm{SNR}=E / \mathcal{N}_{0}$ is the SNR, with $E$ denoting the signal energy, and $\beta$ is the effective signal bandwidth defined by (5).

\footnotetext{
7 The same average noise power is assumed at each element.

8 Since the effects of the propagation environment, such as the multipath, are not known at the time of TOA estimation, the use of a template signal or a MF impulse response that includes the overall effects of the channel is not usually possible.

9 Refer to [5,23] for the CRLB for TOA estimation in multipath channels.
} 
From (7), it is observed that unlike the RSS estimation, the accuracy of the TOA estimation can be improved by increasing the SNR and/or the effective signal bandwidth. Therefore, for (ultra) wideband systems, TOA estimation can provide very accurate range information.

\subsection{Time Difference of Arrival (TDOA)}

In the absence of synchronization between the target node and the reference nodes, the TDOA estimation can be performed if there is synchronization among the reference nodes [1]. TDOA estimation provides the difference between the arrival times of two signals traveling between the target node and two reference nodes, which determines the position of the target node on a hyperbola, with foci at the two reference nodes, as shown in Fig. 4.

One approach for estimating TDOA is to first estimate TOA for each signal traveling between the target node and a reference node, and then to obtain the difference between the two estimates. Since the target node and the reference nodes are not synchronized, the TOA estimates include a timing offset, which is the same in all estimates as the reference nodes are synchronized, in addition to the time of flight. Therefore, the TDOA estimate can be obtained as

$$
\tau_{\mathrm{TDOA}}=\hat{\tau}_{1}-\hat{\tau}_{2},
$$

where $\hat{\tau}_{i}$, for $i=1,2$, denotes the TOA estimate for the signal traveling between the target node and the $i$ th reference node.

For the TDOA estimates obtained as in (8), the accuracy limits can be deduced from the CRLB expression in Sect. 2.3. Namely, it is concluded that the accuracy of TDOA estimation increases as effective bandwidth and/or SNR increases.

Another approach for TDOA estimation is to perform cross-correlations of the two signals traveling between the target node and the reference nodes, and to calculate the delay corresponding to the largest cross-correlation value. The cross-correlation function is given by Caffery and Stuber [24]

$$
\phi_{1,2}(\tau)=\frac{1}{T} \int_{0}^{T} r_{1}(t) r_{2}(t+\tau) \mathrm{d} t,
$$

where $r_{i}(t)$, for $i=1,2$, represents the signal traveling between the target node and the $i$ th reference node, and $T$ is the observation interval. From (9), the TDOA estimate is calculated as

$$
\tau_{\mathrm{TDOA}}=\arg \max _{\tau}\left|\phi_{1,2}(\tau)\right| .
$$

Although the cross-correlation-based TDOA estimation in (10) performs well for single path channels and white noise models, its performance can degrade considerably over multi-

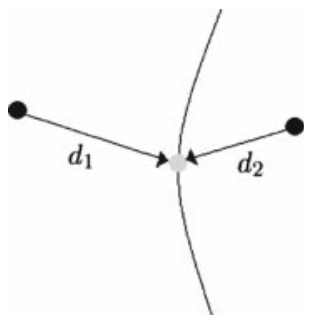

Fig. 4 A TDOA measurement defines a hyperbola passing through the target node with foci at the reference nodes 
path channels and/or colored noise. In order to improve the performance of the cross-correlation scheme in (9) and (10), generalized cross-correlation (GCC) techniques are proposed [25-27]. In GCC-based TDOA estimation, filtered versions of the signals are cross-correlated, which corresponds to shaping the cross-power spectral density of the transmitted signals, in order to provide robustness against colored noise [28].

\subsection{Other Position Related Parameters}

In some positioning systems, two or more of the position related parameters, studied in the previous subsections, can be employed in order to obtain more information about the position of the target node. Examples of such hybrid schemes include TOA/AOA [29], TOA/RSS [30] and TDOA/AOA [31], TOA/TDOA [32] positioning.

In addition to the RSS, AOA and T(D)OA parameters and their combinations, another scheme for position related parameter estimation involves obtaining multipath power delay profile (PDP) or channel impulse response (CIR) related to a received signal [33-36]. In some cases, PDP or CIR estimation can provide significantly more information about the position of the target node than the previously studied schemes. However, extracting the position information from such parameters commonly requires a database consisting of previous PDP (or CIR) estimates. Therefore, algorithms employing PDP or CIR estimation commonly implement training phases, before the actual position estimation process.

Similar to the PDP approach, multipath angular power profile parameter can be estimated at nodes with antenna arrays. Note that both the PDP (CIR) and the angular power profile estimation increase the complexity of the first step in the two-step positioning algorithm in Fig. $1 \mathrm{~b}$ compared to the conventional RSS, AOA, and T(D)OA schemes, since a large number of unknown parameters need to be estimated in the former case. However, such parameters can also facilitate accurate position estimation in challenging environments [36].

\section{Position Estimation}

As shown in Fig. 1b, the second step of a two-step positioning algorithm involves estimation of position from the position related parameters estimated in the first step. Depending on the presence of a database (training data), two types of position estimation techniques can be considered:

- Mapping (fingerprinting) techniques use a database that consists of previously estimated signal parameters at known positions to estimate the position of the target node. Commonly, the database is obtained by a training (off-line) phase before the real-time positioning starts.

- Geometric and statistical techniques do not utilize such a database, and estimate the position of the target node directly from the signal parameters estimated in the first step of the positioning algorithm by using geometric relationships and statistical approaches, respectively.

\subsection{Mapping Techniques}

The main idea behind position estimation via mapping techniques is to determine a regression scheme based on a set of training data, and then to estimate position of a given node according to that regression function. 
Let the training data be expressed as

$$
\mathcal{T}=\left\{\left(\mathbf{m}_{1}, \mathbf{l}_{1}\right),\left(\mathbf{m}_{2}, \mathbf{l}_{2}\right), \ldots\left(\mathbf{m}_{N_{\mathrm{T}}}, \mathbf{l}_{N_{\mathrm{T}}}\right)\right\}
$$

where $\mathbf{l}_{i}$ is the position (location) vector for the $i$ th training data, which is given by $\mathbf{l}_{i}=$ $\left[\begin{array}{ll}x_{i} & y_{i}\end{array}\right]^{T}$ for two-dimensional positioning, $\mathbf{m}_{i}$ represents the vector of estimated parameters for the $i$ th position, and $N_{\mathrm{T}}$ is the total number of elements in the training set (i.e., "size" of the database). Depending on signal parameters employed in the positioning algorithm, $\mathbf{m}_{i}$ consists of a number of position related parameters related to the reference nodes; e.g., each element of $\mathbf{m}_{i}$ can be an RSS estimate at a reference node when the target node is at location $\mathbf{I}_{i}$.

Given the training set in (11), a mapping technique first determines a position estimation rule (pattern matching algorithm/regression function), and then estimates the position $\mathbf{I}$ of a given target node based on a parameter vector $\mathbf{m}$ related to that target node. Some common mapping techniques employed in position estimation include $k$-nearest-neighbor ( $k$-NN) estimation, support vector regression (SVR), and neural networks [36-40].

Due to its simplicity, the $k$-NN estimation technique is considered in this section in order to provide intuition on mapping-based position estimation. In its simplest form, the $k$-NN estimation technique estimates the position of the target node as the position vector in the training set $\mathcal{T}$ corresponding to the parameter vector that has the shortest distance to the given (estimated) parameter vector $\mathbf{m}$. That is, the position is estimated as $\mathbf{l}_{j}$, where

$$
j=\arg \min _{i \in\left\{1, \ldots, N_{T}\right\}}\left\|\mathbf{m}-\mathbf{m}_{i}\right\|
$$

with $\left\|\mathbf{m}-\mathbf{m}_{i}\right\|$ representing the Euclidean distance between $\mathbf{m}$ and $\mathbf{m}_{i}$. This scheme is called the 1-NN, or simply the NN, estimation technique.

In general, the $k$-NN scheme estimates the position of the target node according to the $k$ parameter vectors in $\mathcal{T}$ that have the smallest distances to the given parameter vector $\mathbf{m}$. The position estimate $\hat{\mathbf{l}}$ is obtained by a weighted sum of the positions corresponding to those nearest parameter vectors; i.e.,

$$
\hat{\mathbf{l}}=\sum_{i=1}^{k} w_{i}(\mathbf{m}) \mathbf{l}^{(i)},
$$

where $\mathbf{l}^{(1)}, \ldots, \mathbf{l}^{(k)}$ are the positions corresponding to the $k$ nearest parameter vectors, $\mathbf{m}^{(1)}, \ldots, \mathbf{m}^{(k)}$, to $\mathbf{m}$, and $w_{1}(\mathbf{m}), \ldots, w_{k}(\mathbf{m})$ are the weighting factors for each position. In general, the weights are determined according to the parameter vector $\mathbf{m}$ and the training parameter vectors $\mathbf{m}^{(1)}, \ldots, \mathbf{m}^{(k)}$. Various weighting functions can be employed, as studied in [37]. For example, for the uniform weighting scheme, the position estimate is the sample mean of the positions $\mathbf{l}^{(1)}, \ldots, \mathbf{l}^{(k)}$; i.e.,

$$
\hat{\mathbf{I}}=\frac{1}{k} \sum_{i=1}^{k} \mathbf{l}^{(i)} .
$$

The main advantage of mapping techniques is that they can provide very accurate position estimation in challenging environments with multipath and NLOS propagation. In other words, they have a certain degree of inherent robustness against undesired propagation conditions. However, the main disadvantage is the requirement that the training database should be large enough and representative of the current environment for accurate position estimation. In other words, the database should be updated frequently enough so that the channel 
characteristics in the training and position estimation phases do not differ significantly. Such an update requirement can be very costly for positioning systems operating in dynamic environments, such as for an outdoor positioning system.

\subsection{Geometric and Statistical Techniques}

In the absence of a training database, the position is estimated directly from the position related parameters obtained in the first step of a two-step positioning algorithm. In this case, one can employ either a deterministic approach and estimate the position according to certain geometric relationships, or a statistical approach and try to obtain the most likely position for the target node.

\subsubsection{Geometric Techniques}

Geometric techniques solve for the position of the target node as the intersection of position lines obtained from a set of position related parameters at a number of reference nodes. As studied in Sect.2, an RSS or a TOA parameter defines a circle for the position of the target node; hence three parameter estimates can be used to determine the position via trilateration, as shown in Fig. 5. On the other hand, an AOA parameter defines a straight line passing through the target node and the reference node, as shown in Fig. 3. Therefore, two AOA parameters are sufficient to locate the target node via triangulation as shown in Fig. 6. In the case of TDOA-based positioning, each TDOA parameter determines a hyperbola for the position of the target node. For three reference nodes, two range differences (obtained from TDOA parameters) define two hyperbolas, the intersection of which yields the position of the target node, as shown in Fig. 7, However, the position may not always be determined uniquely depending on the geometrical conditioning of the nodes [1,41].

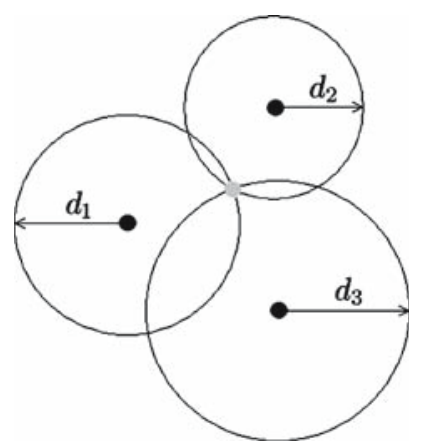

Fig. 5 Determining the position of the target node (the gray node) via trilateration

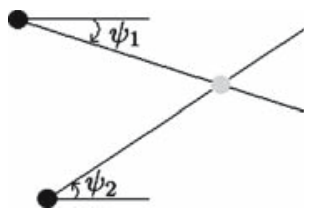

Fig. 6 Determining the position of the target node (the gray node) via triangulation 


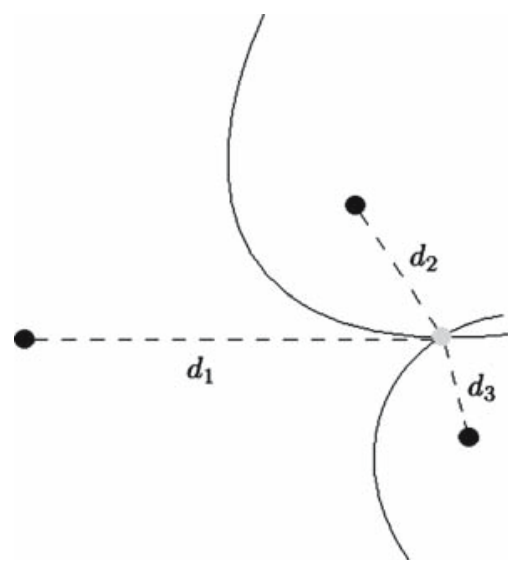

Fig. 7 Positioning via TDOA measurements

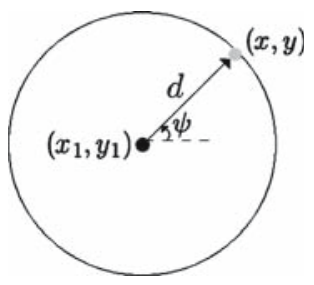

Fig. 8 Hybrid TOA/AOA positioning

The geometric techniques can also be applied to hybrid systems, in which multiple types of position related parameters, such as TDOA/AOA [31] or TOA/TDOA [32], are employed in position estimation. For example, for a hybrid AOA/TOA system as shown in Fig. 8, in which the reference node can estimate both AOA and TOA of the signal from the target node, the position can be calculated as

$$
\begin{aligned}
& x=x_{1}+d \cos \psi, \\
& y=y_{1}+d \sin \psi,
\end{aligned}
$$

where $\left(x_{1}, y_{1}\right)$ is the position of the reference node, $\psi$ the AOA, and $d$ is the range estimate obtained from TOA estimation. In other words, the minimum number of nodes that are required to determine the position of the target node can change depending on the capabilities of the target and/or the reference nodes.

One of the disadvantages of geometric techniques is that they do not provide a theoretical framework in the presence of noise in position related parameters. In other words, when the position lines intersect at multiple points, instead of a single point, due to certain errors in the parameter estimation step, the geometric approach does not provide any inside as to which point to choose as the position of the target node. In addition, as the number of parameters increases, the number of intersections can increase even further. For example, Fig. 9 illustrates three erroneous AOA parameter estimates related to three reference nodes, which results in multiple intersections of the position lines, without all three lines intersecting at a single point. As this example illustrates, the geometric approach does not provide an efficient data fusion mechanism; i.e., cannot utilize multiple parameter estimates efficiently. 


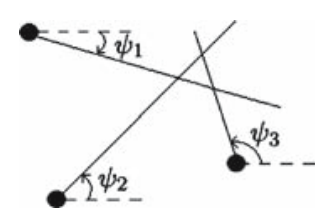

Fig. 9 Position ambiguity in the presence of noisy AOA parameters

\subsubsection{Statistical Techniques}

Unlike the geometric techniques, the statistical approach presents a theoretical framework for position estimation in the presence of multiple position related parameter estimates with or without noise. In order to formulate this generic framework, consider the following model for the parameters estimated in the first step of a two-step positioning algorithm

$$
z_{i}=f_{i}(x, y)+\eta_{i}, \quad i=1, \ldots, N_{\mathrm{m}},
$$

where $N_{\mathrm{m}}$ is the number of parameter estimates, $\eta_{i}$ the noise at the $i$ th estimation, and $f_{i}(x, y)$ is the true value of the $i$ th signal parameter, which is a function of the position of the target, $(x, y)$. Note that $N_{\mathrm{m}}$ is equal to the number of reference nodes for RSS, AOA, and TOA-based positioning, whereas it is one less than the number of reference nodes for TDOA-based positioning as each TDOA parameter is estimated with respect to one reference node.

For various positioning systems, $f_{i}(x, y)$ in (17) can be expressed as ${ }^{10}$

$$
f_{i}(x, y)= \begin{cases}\sqrt{\left(x-x_{i}\right)^{2}+\left(y-y_{i}\right)^{2}}, & \text { TOA/RSS } \\ \tan ^{-1}\left(\frac{y-y_{i}}{x-x_{i}}\right), & \text { AOA }, \\ \sqrt{\left(x-x_{i}\right)^{2}+\left(y-y_{i}\right)^{2}}-\sqrt{\left(x-x_{0}\right)^{2}+\left(y-y_{0}\right)^{2}}, & \text { TDOA }\end{cases}
$$

where $\left(x_{i}, y_{i}\right)$ is the position of the $i$ th reference node and $\left(x_{0}, y_{0}\right)$ is the reference node, relative to which the TDOA parameters are estimated.

In vector notations, the model in (17) can be expressed as

$$
\mathbf{z}=\mathbf{f}(x, y)+\eta,
$$

where $\mathbf{z}=\left[z_{1} \cdots z_{N_{\mathrm{m}}}\right]^{T}, \mathbf{f}(x, y)=\left[f_{1}(x, y) \cdots f_{N_{\mathrm{m}}}(x, y)\right]^{T}$, and $\boldsymbol{\eta}=\left[\eta_{1} \cdots \eta_{N_{\mathrm{m}}}\right]^{T}$.

Depending on the available information related to the noise term $\eta$ in (19), parametric or non-parametric approaches can be followed. In the case that the probability density function of the noise $\boldsymbol{\eta}$ is known except for a set of parameters, denoted by $\lambda$, parametric approaches, such as Bayesian and maximum likelihood (ML) estimation, can be employed. In the absence of information about the form of the probability density function of $\eta$, non-parametric techniques need to be used. Note that the $k$-NN, SVR and neural networks approaches studied in Sect. 3.1 are examples of non-parametric estimators since they do not assume any form for the probability density function of the noise. However, they utilize a training database. Although the form of the density function is unknown in the non-parametric case, there can still be some generic information about some of its parameters [42], such as its variance and symmetry properties, which can be used to design non-parametric estimation rules, such as the least median of squares technique in [43], the residual weighting algorithm in [44], and the variance weighted least squares technique in [45].

10 Time parameters are converted to distance values by scaling by the speed of light. 
For the remainder of this section, the parametric approaches are studied in detail. Let the vector of unknown parameters be represented by $\boldsymbol{\theta}$, which consists of the position of the target node, as well as the unknown parameters of the noise distribution; ${ }^{11}$ i.e., $\boldsymbol{\theta}=\left[\begin{array}{lll}x & y & \lambda^{T}\end{array}\right]^{T}$. Depending on the availability of prior information on $\boldsymbol{\theta}$, Bayesian or ML estimation techniques can be employed [46].

In the presence of prior information on $\boldsymbol{\theta}$, which is represented as a prior probability distribution $\pi(\boldsymbol{\theta})$, the Bayesian approach can be used to obtain an estimate of $\boldsymbol{\theta}$ that minimizes a specific cost function [21]. Two common Bayesian estimators are the minimum mean square error (MMSE) and the maximum a posteriori (MAP) estimators, ${ }^{12}$ which estimate $\boldsymbol{\theta}$ as

$$
\begin{aligned}
\hat{\boldsymbol{\theta}}_{\mathrm{MMSE}} & =\mathrm{E}\{\boldsymbol{\theta} \mid \mathbf{z}\}, \\
\hat{\boldsymbol{\theta}}_{\mathrm{MAP}} & =\arg \max _{\boldsymbol{\theta}} p(\mathbf{z} \mid \boldsymbol{\theta}) \pi(\boldsymbol{\theta}),
\end{aligned}
$$

where $\mathrm{E}\{\boldsymbol{\theta} \mid \mathbf{z}\}$ is the conditional expectation of $\boldsymbol{\theta}$ given $\mathbf{z}$, and $p(\mathbf{z} \mid \boldsymbol{\theta})$ represents the probability density function of $\mathbf{z}$ conditioned on $\boldsymbol{\theta}$.

In the absence of prior information on $\boldsymbol{\theta}$, the ML estimation is commonly employed, which calculates the value of $\boldsymbol{\theta}$ that maximizes the likelihood function; i.e.,

$$
\hat{\boldsymbol{\theta}}_{\mathrm{ML}}=\arg \max _{\boldsymbol{\theta}} p(\mathbf{z} \mid \boldsymbol{\theta}) .
$$

Note that since $\mathbf{f}(x, y)$ is a deterministic function, the likelihood function can be expressed as

$$
p(\mathbf{z} \mid \boldsymbol{\theta})=p_{\eta}(\mathbf{z}-\mathbf{f}(x, y) \mid \boldsymbol{\theta}),
$$

where $p_{\eta}(\cdot \mid \boldsymbol{\theta})$ represents the conditional probability density function of the noise vector conditioned on $\boldsymbol{\theta}$.

Depending on the properties of the noise vector, various scenarios can be considered.

Case 1 Independent Noise Components: For independent noise components, the likelihood function in (23) can be expressed as

$$
p(\mathbf{z} \mid \boldsymbol{\theta})=\prod_{i=1}^{N_{\mathrm{m}}} p_{\eta_{i}}\left(z_{i}-f_{i}(x, y) \mid \boldsymbol{\theta}\right),
$$

where $p_{\eta_{i}}(\cdot \mid \boldsymbol{\theta})$ represents the conditional probability density function for the $i$ th noise component given $\boldsymbol{\theta}$.

The independent noise assumption is usually valid for TOA, RSS and AOA estimation. However, for TDOA estimation, the noise components are correlated, as all TDOAs are computed with respect to the same reference node. Therefore, TDOA-based systems can be studied through the generic expression in (23), or by using a correlated Gaussian model under certain conditions, as will be investigated later.

For TOA, RSS, and AOA based systems in LOS conditions, the parameters of the noise can be assumed to be known, which reduces the unknown parameter vector to $\boldsymbol{\theta}=\left[\begin{array}{ll}x & y\end{array}\right]^{T}$. Also, it is possible to (approximately) model each noise component by a zero mean Gaussian

\footnotetext{
11 In general, the noise components may also depend on the position of the mobile, in which case $\boldsymbol{\theta}$ includes the union of the elements in $\lambda, x$, and $y$.

12 The MAP estimator is not a regular Bayesian estimator, but it fits within the Bayesian framework [21].
} 
random variable in the LOS case [10]; that is,

$$
p_{\eta_{i}}(n)=\frac{1}{\sqrt{2 \pi} \sigma_{i}} \exp \left(-\frac{n^{2}}{2 \sigma_{i}^{2}}\right) .
$$

Then, the likelihood function in (24) can be expressed as

$$
p(\mathbf{z} \mid \boldsymbol{\theta})=\frac{1}{(2 \pi)^{N_{\mathrm{m}} / 2} \prod_{i=1}^{N_{\mathrm{m}}} \sigma_{i}} \exp \left(-\sum_{i=1}^{N_{\mathrm{m}}} \frac{\left(z_{i}-f_{i}(x, y)\right)^{2}}{2 \sigma_{i}^{2}}\right) .
$$

From (26), the ML estimator in (22) can be obtained as

$$
\hat{\boldsymbol{\theta}}_{\mathrm{ML}}=\arg \min _{\left[\begin{array}{ll}
x & y
\end{array}\right]^{T}} \sum_{i=1}^{N_{\mathrm{m}}} \frac{\left(z_{i}-f_{i}(x, y)\right)^{2}}{\sigma_{i}^{2}},
$$

which is the well-known non-linear least-squares (NLS) estimator [1]. Note that the weights are inversely proportional to the noise variances since a larger variance means a less reliable estimate. Common techniques for solving (27) include gradient descent algorithms and linearization techniques via the Taylor series expansion $[1,47]$.

For NLOS conditions between the target node and some reference nodes, the noise model can be significantly different for the parameter estimates at those reference nodes compared to the ones at the LOS nodes. If the positions of the reference nodes are sufficiently separated, the conditional independence assumption in (24) can still hold. Therefore, the ML position estimator can be derived from (22) and (24) by using appropriate noise distributions for LOS and NLOS reference nodes. ${ }^{13}$

The noise distribution in the NLOS case is commonly modeled as the sum of two noise terms, one related to the background noise, and the other related to the NLOS error. In this case, the noise distribution is usually considerably different than the Gaussian model in (25). Some common models for the NLOS error include Gamma distribution [10] and distributions based on certain scattering models [51]. In many cases, the errors due to NLOS propagation dominate the estimation errors due to background noise.

Case 2 Correlated Gaussian Noise Components: For a noise vector modeled as a multivariate Gaussian random variable with mean $\boldsymbol{\mu}$ and covariance matrix $\boldsymbol{\Sigma}$, the likelihood function is given by

$$
p(\mathbf{z} \mid \boldsymbol{\theta})=\frac{1}{(2 \pi)^{N_{\mathrm{m}} / 2}|\mathbf{\Sigma}|^{1 / 2}} \exp \left\{-\frac{1}{2}(\mathbf{z}-\mathbf{f}(x, y)-\boldsymbol{\mu})^{T} \boldsymbol{\Sigma}^{-1}(\mathbf{z}-\mathbf{f}(x, y)-\boldsymbol{\mu})\right\} .
$$

Then, the ML position estimator can be calculated as

$$
\hat{\boldsymbol{\theta}}_{\mathrm{ML}}=\arg \min _{\boldsymbol{\theta}}(\mathbf{z}-\mathbf{f}(x, y)-\boldsymbol{\mu})^{T} \boldsymbol{\Sigma}^{-1}(\mathbf{z}-\mathbf{f}(x, y)-\boldsymbol{\mu})+\log |\boldsymbol{\Sigma}|,
$$

where $\boldsymbol{\theta}$ consists of the position of the target node and the unknown parameters related to $\boldsymbol{\mu}$ and $\boldsymbol{\Sigma}$.

For a noise distribution with zero mean and a known covariance matrix, (29) simplifies to

$$
\hat{\boldsymbol{\theta}}_{\mathrm{ML}}=\arg \min _{\left[\begin{array}{c}
x \\
y
\end{array}\right]^{T}}(\mathbf{z}-\mathbf{f}(x, y))^{T} \boldsymbol{\Sigma}^{-1}(\mathbf{z}-\mathbf{f}(x, y)),
$$

which is called the weighted LS (WLS) solution [1].

13 It is assumed to be known which nodes are LOS and which are NLOS. Such an information can be obtained using NLOS detection algorithms [48-50]. 
Although the independent noise model in Case 1 is not well-suited for TDOA-based positioning systems, the correlated Gaussian noise model in (28) can represent such systems quite accurately for sufficiently large SNRs. As an example, consider TDOA estimation via difference of TOA estimates. If the estimates of range differences (equivalently, TDOA estimates) are modeled as

$$
z_{i}=d_{i}-d_{0}+n_{i}-n_{0}, \quad i=1, \ldots, N_{\mathrm{m}},
$$

where $d_{j}=\sqrt{\left(x-x_{j}\right)^{2}+\left(y-y_{j}\right)^{2}}$ for $j=0,1, \ldots, N_{\mathrm{m}}$, and $n_{0}, n_{1}, \ldots, n_{N_{\mathrm{m}}}$ are zero mean independent Gaussian random variables with variances $\sigma_{0}^{2}, \sigma_{1}^{2}, \ldots, \sigma_{N_{\mathrm{m}}}^{2}$, respectively, then the estimates can be modeled as

$$
\mathbf{z}=\mathbf{f}(x, y)+\eta,
$$

where $f_{i}(x, y)=d_{i}-d_{0}$ for $i=1, \ldots, N_{\mathrm{m}}$, and $\eta \sim \mathcal{N}(\mathbf{0}, \boldsymbol{\Sigma})$ with

$$
\boldsymbol{\Sigma}=\left[\begin{array}{cccc}
\sigma_{1}^{2}+\sigma_{0}^{2} & \sigma_{0}^{2} & \cdots & \sigma_{0}^{2} \\
\sigma_{0}^{2} & \sigma_{2}^{2}+\sigma_{0}^{2} & \ddots & \vdots \\
\vdots & \ddots & \ddots & \sigma_{0}^{2} \\
\sigma_{0}^{2} & \cdots & \sigma_{0}^{2} & \sigma_{N_{\mathrm{m}}}^{2}+\sigma_{0}^{2}
\end{array}\right] .
$$

In other words, the noise is Gaussian with correlated components (since the correlation matrix is not diagonal) in this case.

In the case of NLOS propagation between the target node and a number of reference nodes, the Gaussian model in (28) may not be very accurate. Therefore, the generic ML estimation in (22) and (23) should be performed for TDOA estimation in NLOS scenarios.

\subsection{CRLBs for Position Estimation}

In this section, CRLBs for various position estimation algorithms are investigated. The CRLB for an unbiased estimate $\hat{\boldsymbol{\theta}}$ of $\boldsymbol{\theta}$ can be expressed as

$$
\mathrm{E}\left\{(\hat{\boldsymbol{\theta}}-\boldsymbol{\theta})(\hat{\boldsymbol{\theta}}-\boldsymbol{\theta})^{T}\right\} \geq \mathbf{I}_{\boldsymbol{\theta}}^{-1},
$$

where $\mathbf{I}_{\theta}$ is the FIM given by

$$
\mathbf{I}_{\boldsymbol{\theta}}=\mathrm{E}\left\{\frac{\partial \log p(\mathbf{z} \mid \boldsymbol{\theta})}{\partial \boldsymbol{\theta}}\left(\frac{\partial \log p(\mathbf{z} \mid \boldsymbol{\theta})}{\partial \boldsymbol{\theta}}\right)^{T}\right\}
$$

with $p(\mathbf{z} \mid \boldsymbol{\theta})$ representing the conditional probability density function of the position related parameter estimate given $\boldsymbol{\theta}$, and $\mathbf{I}_{1} \geq \mathbf{I}_{2}$ meaning that $\mathbf{I}_{1}-\mathbf{I}_{2}$ is positive semi-definite.

The CRLB provides also a lower bound on the mean square error (MSE) of a position estimate as follows:

$$
\begin{aligned}
\left.\mathrm{MSE} \triangleq \mathrm{E}\{\| \hat{\boldsymbol{\theta}}-\boldsymbol{\theta}) \|^{2}\right\} & =\operatorname{trace}\left[\mathrm{E}\left\{(\hat{\boldsymbol{\theta}}-\boldsymbol{\theta})(\hat{\boldsymbol{\theta}}-\boldsymbol{\theta})^{T}\right\}\right] \\
& \geq \operatorname{trace}\left[\mathbf{I}_{\boldsymbol{\theta}}^{-1}\right] \triangleq \mathrm{MMSE},
\end{aligned}
$$

where MMSE refers to minimum MSE. In the following, the theoretical limits are investigated in terms of MMSE expressions for TOA, TDOA, RSS, and AOA-based positioning systems. 


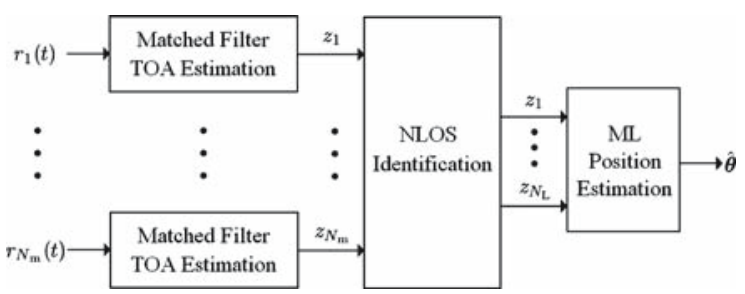

Fig. 10 An asymptotically optimal positioning receiver in the absence of statistical NLOS information

Consider a system with $N_{\mathrm{m}}$ reference nodes, $N_{\mathrm{L}}$ of which are in LOS with the target node, and the remaining ones are in NLOS. Without loss of generality, the LOS and the NLOS nodes are indexed by $i=1, \ldots, N_{\mathrm{L}}$ and $i=N_{\mathrm{L}}+1, \ldots, N_{\mathrm{m}}$, respectively. It is assumed that signals propagate via a single LOS or NLOS path, ${ }^{14}$ and there is no prior statistical information related to errors due to NLOS propagation. Let $\psi_{i}=\tan ^{-1}\left(\frac{y-y_{i}}{x-x_{i}}\right)$, for $i=1, \ldots, N_{\mathrm{m}}$, denote the angle between the $i$ th reference node and the target node, where $(x, y)$ and $\left(x_{i}, y_{i}\right)$ are the positions of the target node and the $i$ th reference node, respectively.

It can be shown that the MMSE for TOA-based positioning is given by Qi et al. [8]

$$
\operatorname{MMSE}_{\mathrm{TOA}}=\frac{c^{2} \sum_{i=1}^{N_{\mathrm{L}}} \sigma_{i}^{-2}}{\sum_{i=1}^{N_{\mathrm{L}}} \sum_{j=1}^{i-1} \sigma_{i}^{-2} \sigma_{j}^{-2} \sin ^{2}\left(\psi_{i}-\psi_{j}\right)},
$$

where $c$ is the speed of light and $\sigma_{i}^{2}$, for $i=1, \ldots, N_{\mathrm{L}}$, is the variance of the zero mean Gaussian noise in the LOS case. Note that the theoretical lower bound in (38) is independent of the parameter estimates related to the NLOS nodes, which means that the NLOS nodes do not contribute to the positioning accuracy in the absence of prior statistical information related to NLOS errors. In addition, the geometric configuration of the LOS nodes can affect the theoretical limit significantly through the last term in the denominator.

For sufficiently large SNR and/or effective bandwidth $\beta, \sigma_{i}^{-2}$ is approximately equal to $8 \pi^{2} \beta^{2} \mathrm{SNR}_{i}$, where $\mathrm{SNR}_{i}$ is the $\mathrm{SNR}$ for the $i$ th signal [10]. In that case (38), can be expressed as

$$
\operatorname{MMSE}_{\mathrm{TOA}}=\frac{c^{2}}{8 \pi^{2} \beta^{2}} \frac{\sum_{i=1}^{N_{\mathrm{L}}} \mathrm{SNR}_{i}}{\sum_{i=1}^{N_{\mathrm{L}}} \sum_{j=1}^{i-1} \mathrm{SNR}_{i} \mathrm{SNR}_{j} \sin ^{2}\left(\psi_{i}-\psi_{j}\right)},
$$

which shows the impact of the effective bandwidth on the MMSE. In [8], it is shown that an ML estimator based on LOS delay estimates obtained via matched filtering (or correlation) can attain the MMSE value in (39) for sufficiently large SNR and/or effective bandwidth. In other words, the two-step positioning receiver in Fig. 10 is asymptotically optimal.

As an example, consider the positioning scenario in Fig. 11, where the target node is surrounded by six reference nodes that are uniformly located on a circle. Assuming that all the signals have the same SNR, the theoretical lower bounds can be obtained as in Fig. 12, where the square root of the MMSE expression in (39) is plotted against the effective bandwidth for various numbers of NLOS nodes. Namely, for $N_{\mathrm{L}}=3$, nodes $4-6$; for $N_{\mathrm{L}}=4$, nodes 5 and 6; and for $N_{\mathrm{L}}=5$, node 6 are the NLOS nodes. From the figure, it is observed that as the number of LOS nodes, the effective bandwidth and/or the SNR increases, the accuracy of the positioning system increases.

14 Refer to [52] for theoretical limits of position estimation in multipath channels. 


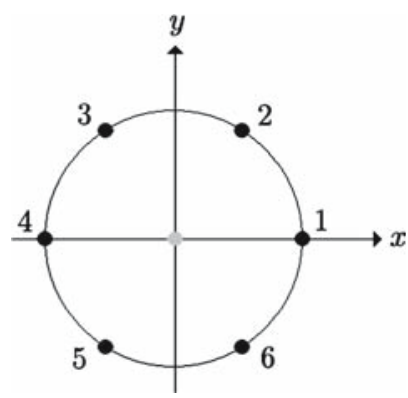

Fig. 11 A positioning scenario, in which six reference nodes are estimating the position of the target node in the middle via TOA estimation

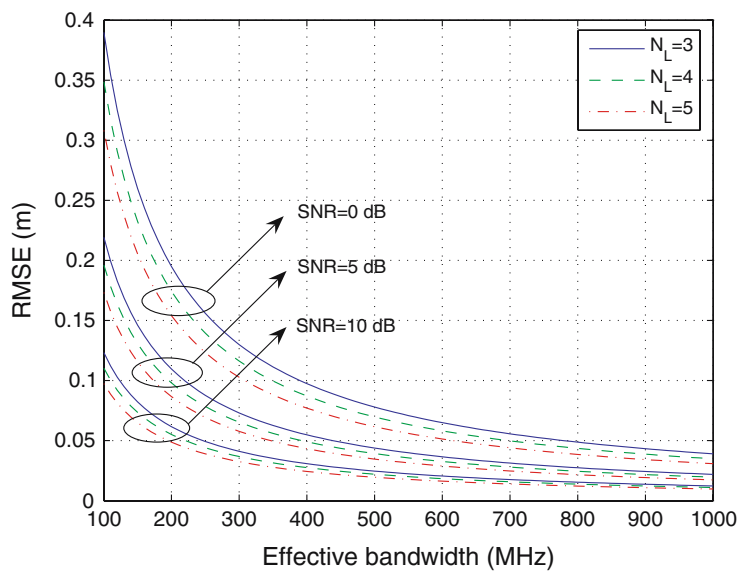

Fig. 12 Square root of the MMSE expression in (39), called RMSE, versus the effective bandwidth for various numbers of NLOS nodes and SNRs. For $N_{\mathrm{L}}=3$, nodes 4-6; for $N_{\mathrm{L}}=4$, nodes 5 and 6; and for $N_{\mathrm{L}}=5$, node 6 are the NLOS nodes

For TDOA-based positioning, the MMSE can be expressed as [8]

$$
\begin{aligned}
\operatorname{MMSE}_{\mathrm{TDOA}}= & c^{2} \sum_{i=1}^{N_{\mathrm{L}}} \sigma_{i}^{-2} \\
& \times \frac{\left(\sum_{i=1}^{N_{\mathrm{L}}} \sigma_{i}^{-2}\right)^{2}-\sum_{i=1}^{N_{\mathrm{L}}} \sigma_{i}^{-4}-\sum_{i=1}^{N_{\mathrm{L}}} \sum_{j=1}^{i-1} \sigma_{i}^{-2} \sigma_{j}^{-2} \cos \left(\psi_{i}-\psi_{j}\right)}{\sum_{i=1}^{N_{\mathrm{L}}} \sum_{j=1}^{i-1} \sigma_{i}^{-2} \sigma_{j}^{-2} K_{i, j}^{2}}
\end{aligned}
$$

where $K_{i, j}$ is given by

$$
K_{i, j}=\sin \left(\psi_{i}-\psi_{j}\right) \sum_{k=1}^{N_{\mathrm{L}}} \sigma_{k}^{-2}+\sum_{k=1}^{N_{\mathrm{L}}} \sigma_{k}^{-2} \sin \left(\psi_{i}-\psi_{k}\right)+\sum_{k=1}^{N_{\mathrm{L}}} \sigma_{k}^{-2} \sin \left(\psi_{j}-\psi_{k}\right) .
$$

As can be observed from (40), the MMSE is independent of the estimates related to NLOS nodes as in the TOA case. Also, it can be shown that the MMSE for TDOA-based positioning 
is always larger than or equal to that for TOA-based positioning [53], which is expected due to the presence of an extra unknown parameter, timing offset, in TDOA systems.

For RSS-based positioning systems, the MMSE is expressed as [8]

$$
\operatorname{MMSE}_{\mathrm{RSS}}=\left(\frac{\ln 10}{10 n}\right)^{2} \frac{\sum_{i=1}^{N_{\mathrm{m}}} \sigma_{\mathrm{sh}, i}^{-2} d_{i}^{-2}}{\sum_{i=1}^{N_{\mathrm{m}}} \sum_{j=1}^{i-1} \sigma_{\mathrm{sh}, i}^{-2} \sigma_{\mathrm{sh}, j}^{-2} d_{i}^{-2} d_{j}^{-2} \sin ^{2}\left(\psi_{i}-\psi_{j}\right)},
$$

where $n$ is the path loss exponent, $\sigma_{\mathrm{sh}, i}^{2}$ is the variance of the log-normal shadowing for the $i$ th signal, and $d_{i}=\sqrt{\left(x-x_{i}\right)^{2}+\left(y-y_{i}\right)^{2}}$ is the distance between the target node and the $i$ th reference node. Note that the accuracy of RSS-based positioning depends heavily on the channel parameters, namely the path loss exponent and the shadowing variances. Also, the accuracy depends on estimates at all nodes, LOS and NLOS, since the effects of NLOS propagation are implicitly included in the RSS signal model, as studied in Sect.2.1.

For an AOA-based positioning system employing ULAs, the MMSE can be expressed as [8]

$$
\begin{aligned}
\operatorname{MMSE}_{\mathrm{AOA}}= & \frac{3}{4 \pi^{2} \Delta^{2} N_{\mathrm{a}}\left(N_{\mathrm{a}}+1\right)\left(2 N_{\mathrm{a}}+1\right)} \\
& \times \frac{\sum_{i=1}^{N_{\mathrm{L}}} \frac{\mathrm{SNR}_{i}}{d_{i}^{2}} \sin ^{2} \psi_{i}}{\sum_{i=1}^{N_{\mathrm{L}}} \sum_{j=1}^{i-1} \frac{\mathrm{SNR}_{i} \mathrm{SNR}_{j}}{d_{i}^{2} d_{j}^{2}} \sin ^{2}\left(\psi_{i}-\psi_{j}\right) \sin ^{2} \psi_{i} \sin ^{2} \psi_{j}},
\end{aligned}
$$

where $N_{\mathrm{a}}$ is the number of antenna elements and $\Delta$ is the inter-element spacing. Similar to the time-based systems, the AOA-based positioning utilizes the estimates from LOS nodes only.

For hybrid positioning systems, two different categories can be considered depending on whether estimation errors for various types of position related parameters are correlated or not. Some lower bound expressions for such systems can be found in [8,30].

The analysis in this section assumes that there is no prior statistical information related to NLOS errors. In the presence of such prior information, the accuracy can be evaluated by means of generalized CRLB (G-CRLB), as investigated in [8]. In that case, an asymptotically optimal positioning receiver can be implemented as shown in Fig. 13. Note that estimates from both LOS and NLOS reference nodes are utilized in the presence of NLOS error statistics.

\section{Concluding Remarks}

Various positioning algorithms have been investigated and theoretical limits for their positioning accuracy have been presented in terms of CRLBs. A two-step approach to position estimation has been adopted. First, estimation of position related parameters has been studied and accuracy of RSS, AOA, and T(D)OA estimation has been quantified in terms of CRLBs. Then, for the second step, estimation of position based on position related parameters estimated in the first step has been studied, and mapping, geometric and statistical approaches have been investigated.

Note that the position estimation schemes considered in this paper have been based on a single observation of signals at a given time instead of multiple observations over a period of time. For the latter, tracking algorithms, such as Kalman filters, grid-based approaches or particle filters can be employed [54]. 


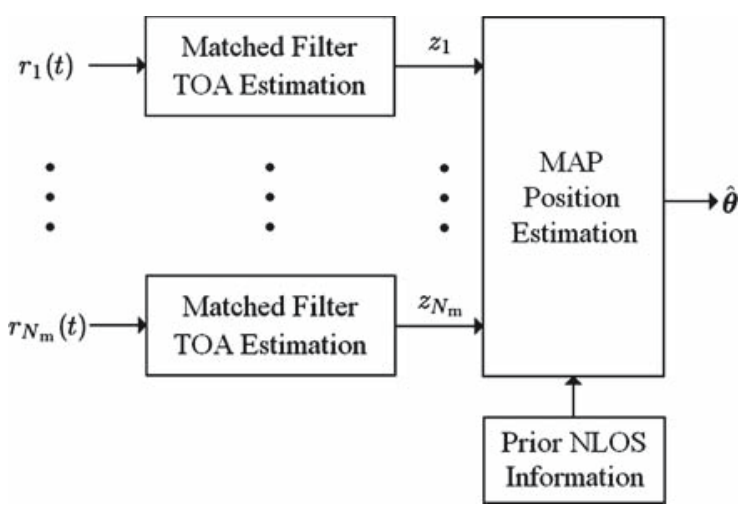

Fig. 13 An asymptotically optimal receiver for TOA-based positioning in the presence of statistical NLOS information

\section{References}

1. Caffery, J. J. (2000). Wireless location in CDMA cellular radio systems. Boston: Kluwer.

2. "IEEE 15-03-0489-03-004a-application-requirement-analysis-031127 v0.4." [Online]. Available: http:// www.ieee802.org/15/pub/TG4.html

3. IEEE P802.15.4a/D4 (Amendment of IEEE Std 802.15.4), "Part 15.4: Wireless medium access control (MAC) and physical layer (PHY) specifications for low-rate wireless personal area networks (LRWPANs)," July 2006.

4. Commission, F. C. (1996). Revision of the commissions rules to insure compatibility with enhanced 911 emergency calling systems. FCC Docket No. 94-102.

5. Gezici, S., Tian, Z., Giannakis, G. B., Kobayashi, H., Molisch, A. F., Poor, H. V., \& Sahinoglu, Z. (2005). Localization via ultra-wideband radios: A look at positioning aspects for future sensor networks. IEEE Signal Processing Magazine, 22(4), 70-84.

6. Gustafsson, F., \& Gunnarsson, F. (2005). Mobile positioning using wireless networks. IEEE Signal Processing Magazine, 22(4), 41-53.

7. Weiss, A. J. (2004). Direct position determination of narrowband radio frequency transmitters. IEEE Signal Processing Letters, 11(5), 513-516.

8. Qi, Y., Kobayashi, H., \& Suda, H. (2006). Analysis of wireless geolocation in a non-line-of-sight environment. IEEE Transactions on Wireless Communications, 5(3), 672-681.

9. Proakis, J. G. (2000). Digital communications (4th ed.). New York: Mc Graw Hill.

10. Qi, Y. (2004). Wireless geolocation in a non-line-of-sight environment. Ph.D. Dissertation, Princeton University.

11. Patwari, N., Ash, J. N., Kyperountas, S., Hero, A. O., Moses, R. L., \& Correal, N. S. (2005). Locating the nodes: Cooperative localization in wireless sensor networks. IEEE Signal Processing Magazine, 22(4), 54-69.

12. Gezici, S., Sahinoglu, Z., Kobayashi, H., \& Poor, H. V. (2005) Ultra wideband geolocation. New York: Wiley, in Ultrawideband Wireless Communications.

13. Mallat, A., Louveaux, J., \& Vandendorpe, L. (2007). UMB based positioning in multipath channels: CRBS for AOA and for hybrid TOA-AOA based methods. In: Proceedings of the IEEE international conference on communications (ICC), Glasgow, Scotland, June 2007.

14. Lee, J.-Y., \& Scholtz, R. A. (2002). Ranging in a dense multipath environment using an UWB radio link. IEEE Journal of Selected Areas Communications, 20(9), 1677-1683.

15. Lindsey, W. C., \& Simon, M. K. (1991). Phase and Doppler measurements in two-way phase-coherent tracking systems. New York: Dover.

16. Turin, G. L. (1960). An introduction to matched filters. IRE Transactions on Information Theory, IT-6(3), 311-329.

17. Pallas, M.-A., \& Jourdain, G. (1991). Active high resolution time delay estimation for large BT signals. IEEE Transactions on Signal Processing, 39, 781-788.

18. Guvenc, I. \& Sahinoglu, Z. (2005). Threshold-based TOA estimation for impulse radio UWB systems. In Proceedings of the IEEE int. conf. UWB (ICU), Zurich, Switzerland, pp. 420-425. 
19. Gezici, S., Sahinoglu, Z., Kobayashi, H., Poor, H. V., \& Molisch, A. F. (2005). A two-step time of arrival estimation algorithm for impulse radio ultrawideband systems. In Proceedings of the 13th European signal processing conf. (EUSIPCO 2005), Antalya, Turkey.

20. Yang, L., \& Giannakis, G. B. (2004). Blind uwb timing with a dirty template. In Proceedings of the intl. conf. on acoustics, speech and signal processing, Montreal, Quebec, Canada, Vol. 4, pp. 509-512.

21. Poor, H. V. (1994). An introduction to signal detection and estimation. New York: Springer.

22. Cook, C. E., \& Bernfeld, M. (1970). Radar signals: An introduction to theory and applications. New York: Academic Press.

23. Botteron, C., Host-Madsen, A., \& Fattouche, M. (2004). Cramer-rao bounds for the estimation of multipath parameters and mobiles' positions in asynchronous ds-cdma systems. IEEE Transactions on Signal Processing, 52(4), 862-875.

24. Caffery, J. J., \& Stuber, G. L. (1998). Subscriber location in CDMA cellular networks. IEEE Transactions on Vehicular Technology, 47(2), 406-416.

25. Knapp, C., \& Carter, G. (1976). The generalized correlation method for estimation of time delay. IEEE Transactions on Acoustics, Speech, and Signal Processing, 24, 320-327.

26. Champagne, B., Eizenman, M., \& Pasupathy, S. (1989). Exact maximum likelihood time delay estimation. In Proceedings of the international conference of acoustics, speech, and signal processing (ICASSP 1989), Glasgow, Scotland, Vol. 4, pp. 23-26.

27. Belanger, S. P. (1995). Multisensor TDOA estimation in a multipath propagation environment using the em algorithm. In Proceedings of the 29th asilomar conference on signals, systems and computers (ASILOMAR 1995), Pacific Grove, CA, Vol. 2, pp. 1096-1100.

28. Aatique, M. (1997). Evaluation of TDOA techniques for position location in CDMA. Master's thesis, Virginia Polytechnic Institute and State University.

29. Cong, L., \& Zhuang, W. (2002). Hybrid TOA/AOA mobile user location for wideband CDMA cellular systems. IEEE Transactions on Wireless Communications, 1(3), 439-447.

30. Catovic, A., \& Sahinoglu, Z. (2004). The Cramer-Rao bounds of hybrid TOA/RSS and TDOA/RSS location estimation schemes. IEEE Communications Letters, 8, 626-628.

31. Cong, L., \& Zhuang, W. (2002). Hybrid TDOA/AOA mobile user location for wide-band cdma cellular systems. IEEE Transactions on Wireless Communications, 1, 439-447.

32. Reza, R. I. (2000). Data fusion for improved TOA/TDOA position determination in wireless systems. Ph.D. Dissertation, Virginia Tech.

33. Nerguizian, C., Despins, C., \& Affes, S. (2001). Framework for indoor geolocation using an intelligent system. In Proceedings of the 3rd IEEE workshop on wireless LANs, Newton, MA.

34. Triki, M., Slock, D. T. M., Rigal, V., \& Francois, P. (2006) Mobile terminal positioning via power delay profile fingerprinting: Reproducible validation simulations. In Proceedings of the IEEE vehicular technology conference (VTC 2006 Fall), Montreal, Canada.

35. Althaus, F., Troesch, F., \& Wittneben, A. (2005). Uwb geo regioning in rich multipath environment. In Proceedings of the IEEE vehicular technology conference (VTC 2005 Fall), Dallas, TX.

36. Nerguizian, C., Despins, C., \& Affes, S. (2006). Geolocation in mines with an impulse response fingerprinting technique and neural networks. IEEE Transactions on Wireless Communications, 5, 603-611.

37. McGuire, M., Plataniotis, K. N., \& Venetsanopoulos, A. N. (2003). Location of mobile terminals using time measurements and survey points. IEEE Transactions on Vehicular Technology, 52(4), 999-1011.

38. Gezici, S., Kobayashi, H., \& Poor, H. V. (2003). A new approach to mobile position tracking. In Proceedings of the IEEE sarnoff symposium on advances in wired and wireless communications, Ewing, NJ, pp. 204-207.

39. Lin, T.-N., \& Lin, P.-C. (2005). Performance comparison of indoor positioning techniques based on location fingerprinting in wireless networks. In Proceedings of the international conference on wireless networks, communications and mobile computing, Maui, Hawaii, Vol. 2, pp. 1569-1574.

40. Duda, R. O., Hart, P. E., \& Stork, D. G. (2000). Pattern classification (2nd ed.). New York: WileyInterscience.

41. Sayed, A. H., Taroghat, A., \& Khajehnouri, N. (2005). Network-based wireless location. IEEE Signal Processing Magazine, 22(4), 24-40.

42. Cong, L., \& Zhuang, W. (2005). Non-line-of-sight error mitigation in mobile location. IEEE Transactions on Wireless Communications, 4, 560-573.

43. Casas, R., Marco, A., Guerrero, J. J., \& Falco, J. (2006). Robust estimator for non-line-of-sight error mitigation in indoor localization. EURASIP Journal on Applied Signal Processing, 2006, Article ID 43429, 8 pages, doi: 10.1155/ASP/2006/43429.

44. Chen, P. C. (1999). A non-line-of-sight error mitigation algorithm in location estimation. In Proceedings of the IEEE wireless communications and networking conference (WCNC 1999), New Orleans, LA, Vol. 1, pp. 316-320. 
45. Caffery, J. J., \& Stuber, G. L. (1998). Overview of radiolocation in CDMA cellular systems. IEEE Communications Magazine, 36(4), 38-45.

46. Al-Jazzar, S., \& Caffery, J. J. (2002). ML and bayesian toa location estimators for NLOS environments. In Proceedings of the IEEE vehicular technology conference (VTC 2002) Fall, Vancouver, BC, Vol. 2, 1178-1181.

47. Kim, W., Lee, J. G., \& Jee, G. I. (2006). The interior-point method for an optimal treatment of bias in trilateration location. IEEE Transactions on Vehicular Technology, 55(4), 1291-1301.

48. Borras, J., Hatrack, P., \& Mandayam, N. B. (1998). Decision theoretic framework for NLOS identification. In Proceedings of the IEEE vehicular technology conference (VTC 1998), Ottowa, ON, Canada, Vol. 2, pp. 1583-1587.

49. Venkatraman, S., \& Caffery, J. (2002). A statistical approach to non-line-of-sight BS identification. In Proceedings of the 25th international symposium on wireless personal multimedia communications, Honolulu, HI, pp. 296-300.

50. Gezici, S., Kobayashi, H., \& Poor, H. V. (2003). Non-parametric non-line-of-sight identification. In Proceedings of the IEEE 58th vehicular technology conference (VTC 2003 Fall), Orlando, FL, Vol. 4, pp. 2544-2548.

51. Al-Jazzar, S., Caffery, J. J., \& You, H.-R. (2002). A scattering model based approach to NLOS mitigation in TOA location systems. In Proceedings of the IEEE vehicular technology conference (VTC 2002) spring, Birmingham, AL, pp. 861-865.

52. Qi, Y., Kobayashi, H., \& Suda, H. (2006). On time-of-arrival positioning in a multipath environment. IEEE Transactions on Vehicular Technology, 55(5), 1516-1526.

53. Qi, Y., \& Kobayashi, H. (2003). On relation among time delay and signal strength based geolocation methods. In Proceedings of the IEEE global communications conference, San Francisco, CA, Vol. 7, pp. 4079-4083.

54. Arulampalam, S., Maskell, S., Gordon, N., \& Clapp, T. (2002). A tutorial on particle filters for on-line non-linear/non-Gaussian Bayesian tracking. IEEE Transactions on Signal Processing, 50(2), 174-188.

\section{Author Biography}

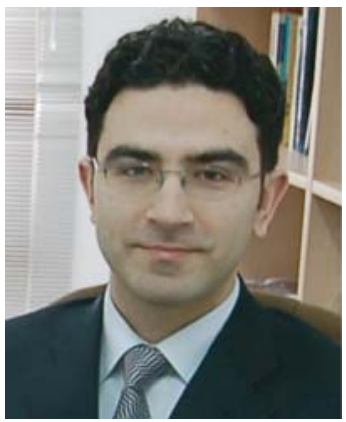

Sinan Gezici received the B.S. degree from Bilkent University, Turkey in 2001, and the Ph.D. degree in Electrical Engineering from Princeton University in 2006. From April 2006 to January 2007, he worked as a Visiting Member of Technical Staff at Mitsubishi Electric Research Laboratories, Cambridge, MA. Since February 2007, he has been an Assistant Professor in the Department of Electrical and Electronics Engineering at Bilkent University.

Dr. Gezici's research interests are in the areas of signal detection, estimation and optimization theory, and their applications to wireless communications and localization systems. Currently, he has a particular interest in ultra-wideband systems for communications and sensing applications. 